\title{
Sensing Behaviour of Some Nanocomposite Systems
}

\author{
Dipankar Chakravorty ${ }^{1}$, Bhola Nath Pal ${ }^{1,2}$, Shilpi Banerjee ${ }^{1}$, Amrita Mandal ${ }^{1,3}$, Sreemanta Mitra ${ }^{1,3}$, \\ Dhriti Ranjan Saha ${ }^{1}$
}

${ }^{1}$ MLS Prof. Unit, Indian Association for the Cultivation of Science, Jadavpur, Kolkata, India; ${ }^{2}$ Nanotechnology and Advanced Spectroscopy, Los Alamos National Laboratory, Los Alamos, USA; ${ }^{3}$ Department of Physics, University of Calcutta, Kolkata, India. Email: mlsdc@iacs.res.in

Received October $1^{\text {st }}, 2013$; revised November $4^{\text {th }}, 2013$; accepted November $11^{\text {th }}, 2013$

Copyright (C) 2013 Dipankar Chakravorty et al. This is an open access article distributed under the Creative Commons Attribution License, which permits unrestricted use, distribution, and reproduction in any medium, provided the original work is properly cited.

\begin{abstract}
Silver nanoparticles of diameters 3.4 to $13.2 \mathrm{~nm}$ grown at the interfaces between silicate glass and some oxide crystallites exhibited about six orders of magnitude reduction in resistivity for a relative humidity change from $25 \%$ to $80 \%$. $\mathrm{Sn}-\mathrm{SnO}_{2}$ nano core-shell structure prepared within a gel-derived silica glass film by electrodeposition technique followed by heat treatment showed large change in resistivity as a function of humidity. The resistivity also changed due to gas flow of $\mathrm{CO}_{2}, \mathrm{C}_{2} \mathrm{H}_{5} \mathrm{OH}$ and $\mathrm{NO}_{2}$, respectively. The latter arose because of reduction/oxidation of $\mathrm{Sn}^{4+} / \mathrm{Sn}^{2+}$ species present at the shell layer of the nanostructures. Nickel nanosheets of thickness $\sim 0.6 \mathrm{~nm}$ grown within the interlayer spaces of Na-4 mica crystallites showed a change of dielectric permittivity (5\%) for an applied magnetic field of 1.2 Tesla. An inhomogeneous model was used to explain this behavior. Two dimensional $\mathrm{CuO}$ phase was grown within the channels of diameter $\sim 5 \mathrm{~nm}$ of mesoporous $\mathrm{SiO}_{2}$ structure. A magnetodielectric (MD) parameter M.D. of $4.4 \%$ was obtained in this case. $\mathrm{BaTiO}_{3}$ nanoparticles of diameter $\sim 25 \mathrm{~nm}$ having pores with diameter $10 \mathrm{~nm}$ showed multiferroic behavior which arose due to the presence of oxygen vacancies as a result of large surface area present. An M.D. parameter of $11 \%$ was found. Similarly mesoporous $\mathrm{LiNbO}_{3}$ of $10 \mathrm{~nm}$ diameter showed an M.D. parameter of $\sim 4.5 \%$ at a magnetic field 1 Tesla. A giant magnetocapacitance effect with a value of $44 \%$ at $1.5 \mathrm{~T}$ was observed in nickel zinc ferrite (NZF) impregnated mesoporous silica. A magnetocapacitance of 51\% at magnetic field $1.7 \mathrm{~T}$ was found in the case of nanocomposites comprising of iron ion containing silica based nanoglass and mesoporous silica. In the last two examples the behavior was explained on the basis of Catalan model of space-charge polarization with extracted values of magnetoresistance of the NZF and nanoglass phases being $58 \%$.
\end{abstract}

Keywords: Nanocomposites; Na-4 Mica; Mesoporous Silica; $\mathrm{BaTiO}_{3}+\mathrm{LiNbO}_{3}$; Magneto Dielectric Effect

\section{Introduction}

Sensing of humidity, gasses of different kinds, magnetic fields etc. have assumed significance in recent time because of the need to control these in the operation of various devices [1-4]. For humidity and gas sensing the surface area of the device becomes important because this controls the reaction with atomic/molecular species thereby changing some of its physical properties. Nanoparticles are characterized by a large surface area per unit volume. We have therefore exploited this strategy to synthesize suitable glass-ceramic-metal nanocomposites for the purpose of humidity and gas sensing. Similarly metal-metal oxide nano core-shell structure was grown within a suitable glass film for sensing humidity as well as some gasses.

Multiferroic systems are ideal for sensing magnetic fields because these exhibit magnetoelectric coupling behavior $[5,6]$. However, such materials are rare in nature and even those reported in the literature posse rather low value of magnetoelectric coupling coefficient [7]. Multiferroic behavior was induced in mesoporous Ba$\mathrm{TiO}_{3}$ and $\mathrm{LiNbO}_{3}$ respectively in which presence of oxygen vacancies introduced uncompensated spins inducing ferromagnetic coupling. On the other hand, heterogeneous nanophase structures exhibit magnetodielectric effent because of Maxwell-Wagner type polarization at the interfaces combined with Hall effect [8]. By a suitable choice of the two phases having different electrical conductivities, a large magnetodielectric effect could be induced.

In this paper, we give a brief description of the work; we have carried out along the lines discussed above. The 
highlights of the results are also given.

\section{Glass-Ceramic-Metal Nanocomposite}

The interfaces between the glass and crystalline phases were used as heterogeneous nucleation sites to grow silver nanoparticles having diameters in the range 3.4 to $13.2 \mathrm{~nm}$. Silicate glasses of suitable compositions and having alkali ion concentrations above $10 \mathrm{~mole} \%$ were prepared by a melt-quench method. The crystalline phases grown were $\mathrm{Zn}_{2} \mathrm{SiO}_{4}, \mathrm{BaTiO}_{3}$ and $\mathrm{LiNbO}_{3}$ respectively. Powdered glass ceramic samples were subjected to an alkali $\leftrightarrow$ silver ion exchange reaction by using a molten bath of silver nitrate. These were then reduced in hydrogen at a suitable temperature. Pellet samples were made by cold pressing of these powders. Electrical measurements were carried out on samples with silver paint electrodes at different humidity levels. A resistivity reduction of four to six orders of magnitude (depending on the nanocomposite system) was observed for a relative humidity change from $25 \%$ to $90 \%$. The absorbed water molecules on the silver nanoparticles gave rise to the localized states and the resistivity was found to arise due to a variable range hopping mechanism. The details have been discussed in reference [9].

\section{3. $\mathrm{Sn}-\mathrm{SnO}_{2}$ Nano Core-Shell Structure in Silica Glass}

$\mathrm{SnO}_{2}-\mathrm{SiO}_{2}$ glass film of suitable composition and a thickness $\sim 5 \mu \mathrm{m}$ was synthesized by sol-gel method on a microscope glass slide. Using two metallic tin electrodes at a separation of $3 \mathrm{~mm}$ and applying a voltage of 30 volts between them metallic channels of tin were generated. The channels were shown to consist of tin nanoparticles with a median diameter $31 \mathrm{~nm}$. By subjecting these films to varying oxidation treatments tin oxide shells of thicknesses ranging from 1.5 to $4.0 \mathrm{~nm}$ were produced. Electrical conduction was shown to be caused by electron hopping between $\mathrm{Sn}^{2+}$ and $\mathrm{Sn}^{4+}$ ions at the core-shell interface. DC resistivity of the films decreased by three orders of magnitude for a relative humidity change from $35 \%$ to $95 \%$. The adsorption of water molecules and the presence of hydroxyl groups and electrons from them formed localized states which lowered the resistivity of the system. The films showed considerable change in resistivity when subjected to different gasses e.g., $\mathrm{CO}$ and $\mathrm{C}_{2} \mathrm{H}_{5} \mathrm{OH}$ pulses over the samples resulted in an increase in electrical conductivity whereas $\mathrm{NO}_{2}$ pulse gave rise to a decrease in the same. In the former case, the gaseous species reduced some of the $\mathrm{Sn}^{4+}$ ions to $\mathrm{Sn}^{2+}$ which increased the number of polarons thus increasing the conductivity. In the latter case, an oxidation reaction increases the number of $\mathrm{Sn}^{4+}$ ions thereby reducing the number of polarons. The details are given in reference
[10].

\section{Ni-Na-4 Mica Nanocomposite}

$\mathrm{Na}_{4} \mathrm{Mg}_{6} \mathrm{Al}_{4} \mathrm{Si}_{4} \mathrm{O}_{20} \mathrm{~F}_{4}, \mathrm{xH}_{2} \mathrm{O}$ commonly referred to as $\mathrm{Na}-4$ mica has an interlayer channel with a thickness of $0.6 \mathrm{~nm}$. Nickel sheets were grown within this space. For this the mica powder was soaked in nickel nitrate solution so that a nickel-enriched layer was formed. The powder was then reduced in $\mathrm{H}_{2}$ at around $1273 \mathrm{~K}$. Nickel nanosheets were produced in the process. Dielectric and magnetodielectric measurements were carried out on silver-coated pellets made by cold pressing of the nanocomposite powder. The dielectric data indicated a dispersion characteristic of a laminar inhomogeneous structure with the layers having different values of electrical conductivity. The dielectric permittivity showed a decrease as a function of the intensity of the applied magnetic field. This could be explained on the basis of space charge polarization at the interface of the two laminae and the Hall effect which deflected some of the charges thereby lowering the value of dielectric permittivity. A 5\% decrease of the latter was observed at a magnetic field of $1.2 \mathrm{~T}$. The details can be found in reference [11].

\section{Mesoporous $\mathrm{BaTiO}_{3}$ and $\mathrm{LiNbO}_{3}$}

To prepare mesoporous $\mathrm{BaTiO}_{3}$ and $\mathrm{LiNbO}_{3}$ a soft template viz., Pluronic P-123 was used. The latter is a copolymer based on poly (ethylene glycol)-poly (propylene glycol)-poly (ethylene glycol). The method consisted of making solutions of this polymer and precursor chemicals for barium and titanium (in case of $\mathrm{BaTiO}_{3}$ ) and lithium and niobium (for $\mathrm{LiNbO}_{3}$ ) separately in suitable solvents. On drying, a gel was produced which after necessary heat treatments resulted in the formation of porous $\mathrm{BaTiO}_{3}$ and $\mathrm{LiNbO}_{3}$ nanoparticles with pore diameters 10 $\mathrm{nm}$ and $4.1 \mathrm{~nm}$, respectively. The surface areas measured were $107 \mathrm{~m}^{2} / \mathrm{g}$ and $185 \mathrm{~m}^{2} / \mathrm{g}$ for $\mathrm{BaTiO}_{3}$ and $\mathrm{LiNbO}_{3}$, respectively. The large surface areas gave rise to large number of oxygen vacancies in these nanoparticles which imparted ferromagnetic behaviour to them bestowing a multiferroic characteristic. Magnetodielectric parameters M.D. $=[\{\varepsilon(H)-\varepsilon(0)\} / \varepsilon(0)] \times 100($ where $\varepsilon(H), \varepsilon(0)$ refer to dielectric constants at magnetic fields $H$ and zero) of $11 \%$ and $4.5 \%$ were measured in $\mathrm{BaTiO}_{3}$ and $\mathrm{LiNbO}_{3}$ at a magnetic field of $1 \mathrm{~T}$. This was explained as caused by the magnetostriction effect of the ferromagnetic component which distorted the crystal lattice giving rise to an increase in the dielectric constant of the material. The details are given in reference $[12,13]$.

\section{CuO-Mesoporous Silica Nanocomposite}

An inhomogeneous structure was created by growing 
$\mathrm{CuO}$ within the two-dimensional hexagonal pores of silica (SBA-15) with a diameter of $5 \mathrm{~nm}$. The latter was prepared by using the copolymer P123 as the template. Tetraethylorthosilicate (TEOS) was used as the precursor chemical and the solid product was obtained after subjecting the solution to a hydrothermal reaction. $\mathrm{CuO}$ was introduced by adding copper nitrate salt solution to the P123 solution containing TEOS. With an increase in the weight percentage of $\mathrm{CuO}$ above about $20 \%$ the mesostructure was found to be destroyed. The composites showed magnetodielectric effect with the M.D. parameter values of $4.4 \%$ and $3 \%$ at magnetic field $1.8 \mathrm{~T}$ recorded for $\mathrm{CuO}$ contents 20 and 35 weight percentages respectively. Parish and Littlewood model was used to explain satisfactorily the variation of the change of the dielectric constant as a function of applied magnetic field. The details of this investigation can be found in reference [14].

\section{Nickel Zinc Ferrite Mesoporous Silica Nanocomposite}

Silica template KIT-6 with a pore size of about $5 \mathrm{~nm}$ was prepared using a technique reported in the literature [15]. The KIT- 6 powder was immersed into a solution of ethanol containing the nitrate salt of nickel, zinc and iron respectively in the proportion needed for producing $\mathrm{Ni}_{0.5} \mathrm{Zn}_{0.5} \mathrm{Fe}_{2} \mathrm{O}_{4}$. The porous silica powder was dried at room temperature and then subjected to a heat treatment at $873 \mathrm{~K}$ for 4 hours. Pellet specimens obtained by cold pressing of the powders were coated with silver paint for the purpose of magnetodielectric measurements. The variation of the real and imaginary parts of dielectric permittivity as a function of frequency at different temperatures indicated a dispersion of the type expected in a system having space charge polarization. This was a direct result of the microstructure which consisted of twodimensional phases $\mathrm{Ni}_{0.5} \mathrm{Zn}_{0.5} \mathrm{Fe}_{2} \mathrm{O}_{4}$ and silica glass having different electrical conductivities. This also resulted in a large magnetodielectric effect with an M.D. parameter of $44 \%$ at room temperature for an applied magnetic field of $1.5 \mathrm{~T}$. These results were explained by Catalan model [16] which describes such a system as a series combination of two capacitors having different dielectric loss factors. By assuming a lowering of electrical resistance of the nanodimensional $\mathrm{Ni}_{0.5} \mathrm{Zn}_{0.5} \mathrm{Fe}_{2} \mathrm{O}_{4}$ phase as a function of applied magnetic field the experimental data could be fitted satisfactorily with the theoretical expression for dielectric permittivity. A negative magnetoresistance of the order of $58 \%$ was extracted from this analysis. The details of the study have been given in reference [17].

\section{Composites of Nanodimensional Silica Glass with Iron Ions and Mesoporous Silica}

These nanocomposites were synthesized by soaking KIT-
6 mesoporous silica pellet in a sol with composition $10 \mathrm{Fe}_{2} \mathrm{O}_{3} \cdot 90 \mathrm{SiO}_{2}$ for 9 hours. The specimen was then subjected to heat treatment at $473 \mathrm{~K}$ for 2 hours. The mesoporous silica had a pore diameter of $5 \mathrm{~nm}$ which was also the thickness of the silica glass synthesized. Chemical analysis showed that there were $\mathrm{Fe}^{2+}$ ions present in the glass the fraction being $\sim 0.01$. This imparted semiconducting characteristics to the nanoglass phase. The nanocomposite exhibited ferromagnetic behaviour though the magnetization was rather small. This was explained as arising due to an antiferromagnetic super exchange interaction between $\mathrm{Fe}^{2+}$ and $\mathrm{Fe}^{3+}$ ions through the intervening oxygen ion. The uncompensated spins gave rise to remanent magnetization. A large magnetodielectric effect was exhibited by these nanocomposites with an M.D. parameter of 51\% for an applied magnetic field of 1.7 tesla. Catalan's model [16] was used to explain this result. For the latter a value of magnetoresistance of the nanoglass phase was extracted as $58 \%$. The details have been described in reference [18].

\section{Conclusion}

Silver particles of diameters in the range of $3.4 \mathrm{~nm}$ to $13.2 \mathrm{~nm}$ grown at the interfaces between silicate glass and $\mathrm{Zn}_{2} \mathrm{SiO}_{4}, \mathrm{BaTiO}_{3}$ and $\mathrm{LiNbO}_{3}$ respectively exhibited about four to six orders of magnitude reduction in resistivity for a relative humidity change from $25 \%$ to $90 \%$. $\mathrm{Sn}-\mathrm{SnO}_{2}$ nano core-shell structure prepared within a silica glass film showed three orders of magnitude resistivity decrease for a relative humidity change from $35 \%$ to $95 \%$. The sample also showed resistivity changes due to gas flow of $\mathrm{CO}, \mathrm{C}_{2} \mathrm{H}_{5} \mathrm{OH}$ and $\mathrm{NO}_{2}$ respectively. This arose because of reduction/oxidation of $\mathrm{Sn}^{4+} / \mathrm{Sn}^{2+}$ species present. Nanocomposites of nickel nanosheets and $\mathrm{Na}-4$ mica showed a change of dielectric constant of $5 \%$ for a magnetic field of $1.2 \mathrm{~T}$. Two dimensional $\mathrm{CuO}$ phase grown within mesoporous $\mathrm{SiO}_{2}$ of pore diameter $5 \mathrm{~nm}$ gave a M.D. parameter of $4.4 \%$. An inhomogeneous conductor model was used to explain these results. Mesoporous $\mathrm{BaTiO}_{3}$ and $\mathrm{LiNbO}_{3}$ nanoparticles showed multiferroic behaviour because of large oxygen vacancies present. These showed magnetodielectric effect with M.D. parameters $11 \%$ and $4.5 \%$ respectively. Giant magnetocapacitance effect was shown by nickel zinc ferrite impregnated mesoporous silica with an M.D. parameter of $44 \%$. Similar effect with an M.D. parameter of $51 \%$ was observed in the case of nanocomposites comprising of iron ion containing silica based on nanoglass and mesoporous silica. The effect in the last two systems was explained on the basis of Catalan model.

\section{Acknowledgements}

The work was supported by Nano Mission Council and 
Indo-Australian project of DST, New Delhi. BNP, SB and DRS thank CSIR and AM, SM thank UGC for the award of Senior Research Fellowship. DC thanks INSA for awarding an honorary scientist position.

\section{REFERENCES}

[1] Y. F. Qiu and S. H. Yang, "ZnO Nanotetrapods: Controlled Vapor-Phase Synthesis and Application for Humidity Sensing," Advanced Functional Materials, Vol. 17, No. 8, 2007, pp. 1345-1352. http://dx.doi.org/10.1002/adfm.200601128

[2] C. L. Dai, M. C. Liu, F. S. Chen, C. C. Wu and M. W. Chang, "A Nanowire $\mathrm{WO}_{3}$ Humidity Sensor Integrated with Micro-Heater and Inverting Amplifier Circuit on Chip Manufactured Using CMOS-MEMS Technique," Sensors and Actuators B: Chemical, Vol. 123, No. 2, 2007, pp. 896-901. http://dx.doi.org/10.1016/j.snb.2006.10.055

[3] C. Wang, L. Yin, L. Zhang, D. Xiang and R. Gao, "Metal Oxide Gas Sensors: Sensitivity and Influencing Factors," Sensors, Vol. 10, No. 3, 2010, pp. 2088-2106. http://dx.doi.org/10.3390/s100302088

[4] D. A. Pan, Y. Bai, Alex A. Volinsky, W. Y. Chu and L. J. Qiao, "Giant Magnetoelectric Effect in Ni-Lead Zirconium Titanate Cylindrical Structure," Applied Physics Letters, Vol. 92, No. 5, 2008, Article ID: 052904-3. http://dx.doi.org/10.1063/1.2841709

[5] R. Ramesh and N. A. Spaldin, "Multiferroics: Progress and Prospects in Thin Films," Nature Materials, Vol. 6, No. 1, 2007, pp. 21-29. http://dx.doi.org/10.1038/nmat1805

[6] C.-W. Nan, M. I. Bichurin, S. Dong, D. Viehland and G. Srinivasan, "Multiferroic Magnetoelectric Composites: Historical Perspective, Status, and Future Directions," Journal of Applied Physics, Vol. 103, No. 3, 2008, Article ID: 031101-34. http://dx.doi.org/10.1063/1.2836410

[7] A. K. Zvezdin, A. M. Kadomtseva, S. S. Krotov, A. P. Pyatakov, Yu. F. Popov and G. P. Vorob'ev, "Magnetoelectric Interaction and Magnetic Field Control of Electric Polarization in Multiferroics," Journal of Magnetism and Magnetic Materials, Vol. 300, No. 1, 2006, pp. 224-228. http://dx.doi.org/10.1016/j.jmmm.2005.10.068

[8] M. M. Parish and P. B. Littlewood, "Magnetocapacitance in Nonmagnetic Composite Media," Physical Review Letters, Vol. 101, No. 16, 2008, Article ID: 166602-4. http://dx.doi.org/10.1103/PhysRevLett.101.166602

[9] B. N. Pal and D. Chakravorty, "Humidity Sensing by Composites of Glass Ceramics Containing Silver Nanoparticles and Their Conduction Mechanism," Sensors and
Actuators B, Vol. 114, No. 2, 2006, pp. 1043-1051. http://dx.doi.org/10.1016/j.snb.2005.07.065

[10] B. N. Pal and D. Chakravorty, "Electrical Properties of Composites with Tin-Tin Oxide Core-Shell Nanostructure and Their Sensing Behaviour," Journal of Physics D: Applied Physics, Vol. 38, No. 18, 2005, pp. 3537-3542. http://dx.doi.org/10.1088/0022-3727/38/18/032

[11] S. Mitra, A. Mandal, A. Datta, S. Banerjee and D. Chakravorty, "Magnetodielectric Effect in Nickel NanosheetNa-4 Mica Composites," Europhysics Letters, Vol. 92, No. 2, 2010, Article ID: 26003-p4. http://dx.doi.org/10.1209/0295-5075/92/26003

[12] S. Banerjee, A. Datta, A. Bhaumik and D. Chakravorty, "Multiferroic Behaviour of Nanoporous $\mathrm{BaTiO}_{3}$," Journal of Applied Physics, Vol. 110, No. 6, 2011, Article ID: 064316-6. http://dx.doi.org/10.1063/1.3641639

[13] S. Banerjee, M. K. Bhunia, A. Bhaumik and D. Chakravorty, "Multifunctional Behaviour of Mesoporous $\mathrm{LiNbO}_{3}$," Journal of Applied Physics, Vol. 111, No. 5, 2012, Article ID: 054310-6. http://dx.doi.org/10.1063/1.3693041

[14] A. Mandal, S. Banerjee, S. Banerjee and D. Chakravorty, "Magnetodielectric Effect of $\mathrm{CuO}$ Grown Within Highly Ordered Two Dimensional Mesoporous Silica Template SBA 15," Journal of Applied Physics, Vol. 112, No. 7, 2012, Article ID: 074310-4. http://dx.doi.org/10.1063/1.4757421

[15] Z. Zhang, F. Zuo and P. Feng, "Hard template synthesis of crystalline mesoporous anatase $\mathrm{TiO} 2$ for photocatalytic hydrogen evolution," Journal of Materials Chemistry, Vol. 20, No. 11, 2010, pp. 2206-2212. http://dx.doi.org/10.1039/b921157h

[16] G. Catalan, "Magnetocapacitance without Magnetoelectric Coupling," Applied Physics Letters, Vol. 88, No. 10, 2006, Article ID: 102902-3. http://dx.doi.org/10.1063/1.2177543

[17] S. Banerjee, P. Hajra, A. Bhaumik and D. Chakravorty, "Giant Magnetocapacitance Effect in Nickel Zinc Ferrite Impregnated Mesoporous Silica," Materials Letters, Vol. 79, 2012, pp. 65-68. http://dx.doi.org/10.1016/j.matlet.2012.03.076

[18] D. R. Saha, S. Banerjee, A. K. Nandi and D. Chakravorty, "Ferromagnetic-Like Behavior in Nano Silica Glass Containing Iron Ions and Giant Magnetodielectric Effect in Composites of these Glasses with Mesoporous Silica," The Journal of Physical Chemistry C, Vol. 116, No. 41, 2012, pp. 21679-21684. http://dx.doi.org/10.1021/jp306017k 